\title{
A BOUNDARY INTEGRAL EQUATION FORMULATION FOR THE HELMHOLTZ EQUATION IN A LOCALLY PERTURBED HALF-PLANE
}

\author{
SIMON N. CHANDLER-WILDE \\ DEPARTMENT OF MATHEMATICS AND STATISTICS, \\ BRUNEL UNIVERSITY, UXBRIDGE, MIDDLESEX, UNITED KINGDOM, UB8 3PH \\ AND ANDREW T. PEPLOW \\ MWL, DEPARTMENT OF AERONAUTICAL \& VEHICLE ENGINEERING, KTH, \\ STOCKHOLM, \\ SWEDEN, S 10044 \\ JUNE 2002
}

\begin{abstract}
In this paper we study the application of boundary integral equation methods to the solution of the Helmholtz equation in a locally perturbed half-plane with Robin or impedance boundary conditions. This problem models outdoor noise propagation from a cutting onto a surrounding flat plane, and also the harbour resonance problem in coastal engineering. We employ Green's theorem to derive a system of three coupled integral equations. The three unknowns are the pressure on the boundary of the disturbance and the pressure and its normal derivative on the interface with the upper half-space. We prove that the integral equation formulation has a unique solution at all wavenumbers by proving equivalence of the boundary value problem and the integral equation formulation and proving uniqueness of solution for the boundary value problem.
\end{abstract}

Key words. Half-plane, Boundary integral equations, Helmholtz equation, uniqueness.

1. Introduction. In this paper a boundary integral equation formulation for the two-dimensional Helmholtz equation in a locally perturbed half-plane is developed to calculate sound propagation out of a cutting of arbitrary cross-section and surface impedance onto surrounding flat rigid or homogeneous impedance ground. Specifically, the case considered is that of propagation from a monofrequency coherent line source in a cutting which is assumed to be straight and infinitely long with crosssection and surface treatment that do not vary along its length. The impedance is allowed to vary in the cutting in the plane perpendicular to the line source so that 
it is possible to model, for example, a road running down the centre of the cutting, with grass banks on either side.

Let $U=\left\{\left(x_{1}, x_{2}\right): x_{1} \in \mathbf{R}, x_{2}>0\right\}$ be the upper half-plane with boundary $\partial U=\left\{\left(x_{1}, 0\right): x_{1} \in \mathbf{R}\right\}$ and let $x^{(1)}=(a, 0), x^{(2)}=(b, 0) \in \partial U$ with $a<b$, $\gamma_{2}:=\left\{\left(x_{1}, 0\right): a \leq x_{1} \leq b\right\}$ and $\gamma_{3}:=\partial U \backslash \gamma_{2}$. Let $\gamma_{1}$ be any simple arc connecting and including $x^{(1)}$ and $x^{(2)}$ which lies entirely (apart from its end-points $x^{(1)}$ and $\left.x^{(2)}\right)$ below the closed upper half-plane $\bar{U}$ and is such that $\gamma_{1} \cup \gamma_{3}$ is an infinite arc of class $C^{2}$. Then $\gamma_{1} \cup \gamma_{3}$ divides the plane into two regions. Let $D$ be the region above $\gamma_{1} \cup \gamma_{3}=\partial D$, containing $U$, and let $S$ be the region enclosed by $\gamma_{1} \cup \gamma_{2}$; see Figure 5.1.

We will discuss the solution of the boundary value problem (BVP) consisting of the Helmholtz equation in the region $D$ with an impedance or Robin boundary condition on $\partial D$ and its reformulation as a boundary integral equation (BIE). We will assume throughout that $\gamma_{3}$ has a constant admittance $\beta_{c}$ with $\beta_{c}=0$ (rigid boundary) or $\Re \beta_{c}>0$ (energy absorbing boundary).

Boundary integral equation formulations for this problem but assuming an entirely rigid boundary (leading to a Neumann boundary condition) are given in the context of predicting water-wave climates in harbours in $[1,2,3]$. The harbour resonance problem is of importance in coastal engineering, where small harbour oscillations excite large motions in ship-mooring causing considerable damage. To minimize such events the characteristics of harbour response must be determined. Hwang and Tuck [1] adopt a single-layer potential method which determines the wave-induced oscillations using a distribution of sources along the boundary of the harbour $\left(\gamma_{1}\right)$ and coastline $\left(\gamma_{3}\right)$ with unknown source strengths. Lee [2] applies Green's second theorem in both the regions inside and outside the harbour, $S$ and $U$, respectively, which is the method adopted in this paper, and matches the wave amplitudes and their normal derivatives at the harbour entrance $\left(\gamma_{2}\right)$. The same integral equation approach as Lee [2] is used by Shaw [3]. These methods were compared with experimental scale 
model measurements for rectangular basins and real harbours and good agreement was found.

Gartmeier [4] applied the integral equation method to the Dirichlet and Neumann problems in the three-dimensional case where the region is a half-space with a local disturbance directed into the medium of propagation. Chandler-Wilde et. al. [5] and Hothersall et. al. [6] consider a similar integral equation formulation for the two-dimensional case with impedance boundary conditions, as a model of outdoor sound propagation over noise barriers on an impedance plane. Peplow et. al. [7] make numerical predictions of sound attenuation, in excess of free-field propagation, for a traffic noise spectrum for a real site where the traffic noise is propagating out of a cutting and onto surrounding flat ground similar to the model used in this study. Proving uniqueness here guarantees non-spurious solutions at all wavenumbers for the numerical solutions in [7].

Willers [8] considers a general local disturbance and writes the solution of the Dirichlet problem in the perturbed half-space in the form of a double-layer potential with a density defined on the whole of the boundary, $\partial D$. An integral equation for this density is found by imposing the Dirichlet data on $\partial D$. Willers reformulates this integral equation as an integral equation with compact integral operator and proves uniqueness and existence of solution for this integral equation and his original BVP formulation. Willers derives a similar BIE formulation for the Neumann problem, but his method does not appear to extend to the case of impedance (Robin) boundary conditions considered here.

Using boundary integral equation or coupled integral equation/finite element methods, various authors $[9,10,11,12]$ have considered the related problem of electromagnetic scattering by an indented perfectly conducting screen. In two dimensions, with transverse magnetic polarization, this electromagnetic problem reduces to the rigid boundary (Neumann condition) case of the problem considered here. In the most recent of these papers, Asvestas and Kleinman [12] derive by an ingenious ar- 
gument a formulation of this electromagnetic problem as a single integral equation on the local perturbation $\gamma_{1}$ (as opposed to the system of three integral equations we derive in this paper). For the case of an entirely rigid boundary their formulation as a single integral equation must be preferred for efficient numerical computation to the system of equations we discuss (with the caveat that Asvestas and Kleinman do not establish that their integral equation is uniquely solvable at all frequencies). However, just as with the formulation of Willers [8], although their formulation applies to both Dirichlet and Neumann boundary conditions, it appears that it is not possible to extend it to the impedance boundary condition case. It must be emphasised here that this study of a coupled system of three integral equations does not include a proof of existence of solutions for the Robin problem. The construction of the proof leads specifically to the uniqueness result. However recent results by Krutitskii [13] and [14] give constructive methods that may lead to solvability. Indeed an entirely different reformulation of the boundary value problem, to the one stated here, may be necessary for a solvability proof.

Section 2 describes the formulation of the boundary value problem in the unbounded domain. It is proved in this section that the boundary value problem has a unique solution at all wavenumbers by a modification of usual arguments employing Green's first theorem. Some known properties needed later of single-layer and doublelayer potentials and of a non-standard modified single-layer potential are summarised in section 3 .

The boundary integral equation is formulated in section 4, derived from the BVP via applications of Green's second theorem in $U$ and $S$. Our formulation coincides with that of Lee [2] when the boundary is completely rigid $\left(\beta_{c}=0\right)$. For $\beta_{c} \neq 0$ we use, as the fundamental solution in $U$, the Green's function for the Helmholtz equation in a half-plane with constant impedance boundary condition.

The integral equation formulation is a coupled system of three integral equations, two second-kind Fredholm equations and one first-kind Fredholm equation. A com- 
mon problem with integral equation formulations in wave scattering is non-uniqueness of solution at a discrete set of irregular wavenumbers: indeed, it appears that Asvestas and Kleinman [12], in their introductory remarks, criticise our formulation, at least in the rigid boundary case, as suffering from precisely this problem at resonances of the region $S$. To the contrary, we establish in section 4 (and this is our main result) equivalence of our integral equation formulation with the original BVP and thus uniqueness of solution of the integral equation formulation at all wavenumbers. Thus the validity of our formulation and that used previously by Lee [2] is established.

Throughout, for $x=\left(x_{1}, x_{2}\right) \in \mathbf{R}^{2}$ let $x^{\prime}=\left(x_{1},-x_{2}\right)$ denote the image of $x$ in $\partial U$. For $G \subset \mathbf{R}^{2}$ let $G^{\prime}:=\left\{x^{\prime}: x \in G\right\}$. Let $B_{R}$ denote the open ball of radius $R$ centred on the origin

2. Formulation as a boundary value problem. For any domain $G$ with boundary $\partial G$ of class $C^{2}$, we introduce the linear space $\mathcal{R}(G)$ of all complex-valued functions $p \in C^{2}(G) \cap C(\bar{G})$ for which the normal derivative on the boundary exists in the sense that the limit

$$
\frac{\partial p}{\partial n}(x)=\lim _{\substack{h \rightarrow 0 \\ h>0}}(n(x) . \nabla p(x-h n(x))), \quad x \in \partial G,
$$

exists uniformly on $\partial G$, where $n(x)$ is the unit normal at $x \in \partial G$ directed out of $G$.

Given a source at $x_{0}$ somewhere in the region $D$, the pressure induced at $x$, denoted by $p(x)$ (a harmonic time dependence $e^{-i w t}$ is assumed and suppressed throughout), may be written as the sum of the incoming field and the scattered field, that is $p(x)=G_{f}\left(x, x_{0}\right)+P(x)$ where $G_{f}\left(x, x_{0}\right):=-\frac{i}{4} H_{0}^{(1)}\left(k\left|x-x_{0}\right|\right)\left(H_{0}^{(1)}\right.$ the Hankel function of the first-kind of order zero and $k$ the wavenumber) is the free-field Green's function.

The pressure $p$ is assumed to satisfy the following boundary value problem.

BVP1 Given $k>0$ and $\beta \in C(\partial D)$ such that $\beta$ is constant $\left(=\beta_{c}\right)$ on $\gamma_{3}$, find $p: \bar{D} \backslash\left\{x_{0}\right\} \rightarrow \mathbf{C}$ such that

$$
p(x)=P(x)+G_{f}\left(x, x_{0}\right), \quad x \in \bar{D} \backslash\left\{x_{0}\right\},
$$


with $P \in \mathcal{R}(D) \cap \mathcal{R}(U) \cap C^{1}\left(\bar{D} \backslash \gamma_{1}\right)$, and such that $p$ satisfies the Helmholtz equation,

$$
\left(\nabla^{2}+k^{2}\right) p(x)=0, \quad x \in D \backslash\left\{x_{0}\right\},
$$

the impedance boundary condition,

$$
\partial p(x) / \partial n=i k \beta(x) p(x), \quad x \in \partial D,
$$

and the Sommerfeld radiation and boundedness conditions,

$$
\begin{aligned}
\partial p(x) / \partial r-i k p(x) & =o\left(r^{-1 / 2}\right), \\
p(x) & =O\left(r^{-1 / 2}\right),
\end{aligned}
$$

uniformly in $x$ as $r:=|x| \rightarrow \infty$.

REMARK 2.1. The regularity assumption that $P \in \mathcal{R}(U)$ is superfluous in that it is not required to prove uniqueness of solution of BVP1 (see Theorem 2.1). It is included in the formulation to aid in deriving the boundary integral equations given below, and with BVP1 as stated we will prove equivalence with the integral equation formulation. The authors suspect that the other assumptions of BVP1 imply that $P \in \mathcal{R}(U)$ but have not been able to prove this for a general $\beta \in C(\partial D)$. However, if $\beta$ is Hölder continuous in neighbourhoods of $x^{(1)}$ and $x^{(2)}$ then local regularity arguments [15] can be used to show that $\nabla P$ is continuous in neighbourhoods of $x^{(1)}$ and $x^{(2)}$. From this and $P \in \mathcal{R}(D)$ it follows that $P \in \mathcal{R}(U)$.

The above boundary value problem (BVP1) has at most one solution by the following modification of usual arguments using Green's first theorem [15].

Theorem 2.1. If $\Re(x) \geq 0, x \in \partial D$, and $\Re \beta_{c}>0$ or $\beta_{c}=0$, then BVP1 has at most one solution.

Proof. Suppose that $p_{1}$ and $p_{2}$ both satisfy BVP1. Then $p:=p_{1}-p_{2} \in \mathcal{R}(D) \cap$ $\mathcal{R}(U) \cap C^{1}\left(\bar{D} \backslash \gamma_{1}\right)$ satisfies the Helmholtz equation (2.2) in $D$, the Robin boundary condition (2.3), and the Sommerfeld radiation and boundedness conditions (2.4). That $p \in \mathcal{R}(D) \cap C^{1}\left(\bar{D} \backslash \gamma_{1}\right)$ and satisfies the Helmholtz equation suffices to guarantee the validity of Green's first theorem applied to $p$ and its conjugate $\bar{p}$ in the domain $B_{R} \cap D$ 
with $R>\max _{x \in \gamma_{2}}|x|$. Thus

$$
\int_{B_{R} \cap D}\left(\bar{p} \nabla^{2} p+|\nabla p|^{2}\right) d S=\int_{\partial\left(B_{R} \cap D\right)} \bar{p} \partial p / \partial n d s .
$$

Since $p$ satisfies the Helmholtz equation, the left hand side is real, so using the boundary condition (2.3) and taking the imaginary part gives

$$
0=k \int_{\partial D \cap B_{R}} \Re(\beta)|p|^{2} d s+k \int_{\partial B_{R} \cap D}|p|^{2} d s+\Im \int_{\partial B_{R} \cap D} \bar{p}(\partial p / \partial r-i k p) d s
$$

By the Sommerfeld radiation and boundedness conditions (2.4) the third term tends to zero as $R \rightarrow \infty$. Since the other terms in equation (2.6) are non-negative it follows that

$$
\int_{\partial D} \Re(\beta)|p|^{2} d s=0
$$

and

$$
\lim _{R \rightarrow \infty} \int_{\partial B_{R} \cap D}|p|^{2}=0
$$

From the first of these equations, by the boundary condition (2.3), it follows that $\partial p / \partial n=0$ on $\partial D \backslash \gamma_{2}$.

Now choose $R_{1}>\max _{x \in \gamma_{2}}|x|$ and define $D_{-}:=D \backslash \bar{B}_{R_{1}}$. Define $p$ in $D_{-}^{\prime}$ by $p(x)=$ $p\left(x^{\prime}\right), x \in D_{-}^{\prime}$. Then $p \in C\left(\mathbf{R}^{2} \backslash \bar{B}_{R_{1}}\right), p$ satisfies the Sommerfeld radiation and boundedness conditions uniformly as $x \rightarrow \infty$ in $\mathbf{R}^{2} \backslash \bar{B}_{R_{1}}$, and $\lim _{R \rightarrow \infty} \int_{\partial B_{R}}|p|^{2} d s=$ 0 .

By Green's representation theorem [15, Theorem 3.3], applied in the regions $D_{-}$ and $D_{-}^{\prime}$,

(2.9) $\int_{\partial D_{-}}\left(p(y) \frac{\partial G_{f}(x, y)}{\partial n(y)}-\frac{\partial p(y)}{\partial n} G_{f}(x, y)\right) d s(y)=\left\{\begin{array}{cc}p(x), & x \in D_{-}, \\ 0, & x \in \mathbf{R}^{2} \backslash \bar{D}_{-},\end{array}\right.$

(2.10) $\int_{\partial D_{\mathcal{\prime}^{\prime}}}\left(p\left(x_{s}\right) \frac{\partial G_{f}(x, y)}{\partial n(y)}-\frac{\partial p(y)}{\partial n} G_{f}(x, y)\right) d s(y)=\left\{\begin{array}{cc}p(x), & x \in D_{\mathcal{-}^{\prime}}, \\ 0, & x \in \mathbf{R}^{2} \backslash \bar{D}_{\mathcal{\prime}^{\prime}},\end{array}\right.$ 
where the normal is directed out of $D_{-}$and $D_{-}{ }^{\prime}$ in the respective equations. Adding the two equations, and since $\partial p / \partial n=0$ on $\partial D \backslash \gamma_{2}$, we obtain

$$
p(x)=\int_{\partial B_{R_{1}}} p(y) \frac{\partial G_{f}(x, y)}{\partial n(y)}-\frac{\partial p(y)}{\partial n} G_{f}(x, y) d s(y), \quad x \in \mathbf{R}^{2} \backslash \bar{B}_{R_{1}} .
$$

Hence $p \in C^{2}\left(\mathbf{R}^{2} \backslash \bar{B}_{R_{1}}\right)$ and satisfies the Helmholtz equation in this region. Since

$$
\lim _{R \rightarrow \infty} \int_{\partial B_{R_{1}}}|p|^{2}=0
$$

from a result due to Rellich [16], $p \equiv 0$ in $\mathbf{R}^{2} \backslash B_{R_{1}}$. By analytic continuation [15, Theorem 3.5], $p \equiv 0$ in $D$.

For future reference a theorem on uniqueness of solution of an exterior Dirichlet problem is also stated. A proof is outlined in Jörgens [18] (and see Colton and Kress $[17])$.

TheOREm 2.2. Let $\Omega$ be an unbounded open connected subset of $\mathbf{R}^{2}$ with a bounded boundary $\partial \Omega$. Suppose that $u \in C^{2}(\Omega) \cap C(\bar{\Omega})$ satisfies

$$
\begin{aligned}
\left(\nabla^{2}+k^{2}\right) u=0 & \text { in } \quad \Omega, \\
u=0 & \text { on } \quad \partial \Omega,
\end{aligned}
$$

and the Sommerfeld radiation and boundedness conditions (2.4). Then $u \equiv 0$ in $\bar{\Omega}$.

3. Single and double-layer potentials. Let $\gamma$ be a closed curve of class $C^{2}$ which encloses a bounded region $\Omega$. Given a function $\phi \in C(\gamma)$, the function

$$
u(x)=S_{\gamma} \phi(x)=\int_{\gamma} G_{f}(x, y) \phi(y) d s(y), \quad x \in \mathbf{R}^{2},
$$

is called the single-layer potential with density $\phi$, and, given $\psi \in C(\gamma)$,

$$
v(x)=K_{\gamma} \psi(x)=\int_{\gamma} \frac{\partial G_{f}(x, y)}{\partial n(y)} \psi(y) d s(y), \quad x \in \mathbf{R}^{2},
$$

is called the double-layer potential with density $\psi$, where $n(y)$ is the normal at $y$ directed out of $\Omega$.

When $x \in \gamma$ the integrals in (3.1) and (3.2) are well-defined as improper integrals [15]. We shall distinguish by subscripts + and - the limits obtained by approaching 
the boundary $\gamma$ from inside $\Omega$ and $\mathbf{R}^{2} \backslash \Omega$, respectively. It is easy to see that $u, v \in$ $C^{\infty}\left(\mathbf{R}^{2} \backslash \gamma\right)$. In the following lemmas the regularity of $u$ and $v$ in the neighbourhood of $\gamma$ is addressed.

Lemma 3.1. [15, Theorem 2.12] The single-layer potential $u$ with continuous density $\phi$ is continuous throughout $\mathbf{R}^{2}$.

Lemma 3.2. [15, Corollary 2.20] For the single-layer potential u with continuous density $\phi$ we have the jump relation

$$
\frac{\partial u_{+}}{\partial n}-\frac{\partial u_{-}}{\partial n}=-\phi \quad \text { on } \gamma,
$$

where

$$
\frac{\partial u_{ \pm}}{\partial n}(x)=\lim _{\substack{h \rightarrow 0 \\ h>0}} \frac{\partial u}{\partial n(x)}(x \mp h n(x)), \quad x \in \gamma,
$$

and the convergence in (3.3) is uniform on $\gamma$.

Lemma 3.3. [15, Theorem 2.13] The double-layer potential $v$ with continuous density $\psi$ can be continuously extended from $\Omega$ to $\bar{\Omega}$ and from $\mathbf{R}^{2} \backslash \bar{\Omega}$ to $\mathbf{R}^{2} \backslash \Omega$ with limiting values

$$
v(x)_{ \pm}=K \psi(x) \pm \frac{1}{2} \psi(x) \quad \text { on } \gamma
$$

where

$$
v_{ \pm}(x):=\lim _{\substack{h \rightarrow 0 \\ h>0}} v(x \mp h n(x)) .
$$

Lemma 3.4. [15, Theorem 2.15] The double-layer potential $v$ with continuous density $\psi$ is continuous on $\gamma$.

Lemma 3.5. [15, Theorem 2.21] For the double-layer potential $v$ with continuous density $\psi$, we have

$$
\frac{\partial v_{+}}{\partial n}=\frac{\partial v_{-}}{\partial n} \quad \text { on } \gamma
$$

in the sense that

$$
\lim _{\substack{h \rightarrow 0 \\ h>0}}\left\{\frac{\partial}{\partial n} v(x+h n(x))-\frac{\partial}{\partial n} v(x-h n(x))\right\}=0, \quad x \in \gamma,
$$


uniformly for $x$ on $\gamma$.

Lemma 3.6. [15, Section 3.2 and Theorem 3.2] The single-layer potential $u$ and the double-layer potential $v$ satisfy the Helmholtz equation (2.2) in $\mathbf{R}^{2} \backslash \gamma$ and the Sommerfeld radiation and boundedness conditions (2.4).

Remark 3.1. Lemmas 3.1, 3.4 and 3.6 are still valid if $\gamma$ is an open arc.

We denote by $G_{\beta_{c}}\left(x, x_{0}\right)$ the fundamental solution of the Helmholtz equation in $\bar{U}$ which satisfies the Sommerfeld radiation and boundedness conditions (2.4) and the impedance boundary condition $\partial G_{\beta_{c}}\left(x, x_{0}\right) / \partial n(x)=i k \beta_{c} G_{\beta_{c}}\left(x, x_{0}\right), x \in \partial U$. $G_{\beta_{c}}\left(x, x_{0}\right)$ may be written as [19]:

$$
G_{\beta_{c}}\left(x, x_{0}\right)=G_{f}\left(x, x_{0}\right)+G_{f}\left(x, x_{0}^{\prime}\right)+\hat{P}_{\beta_{c}}\left(k\left(x-x_{0}^{\prime}\right)\right)
$$

where, for $-\infty<\xi<+\infty, \eta \geq 0$,

$$
\hat{P}_{\beta_{c}}((\xi, \eta)):= \begin{cases}0, & \beta_{c}=0, \\ \frac{i \beta_{c}}{2 \pi} \int_{-\infty}^{+\infty} \frac{\exp \left(i\left(\eta\left(1-s^{2}\right)^{1 / 2}-\xi s\right)\right)}{\left(1-s^{2}\right)^{1 / 2}\left(\left(1-s^{2}\right)^{1 / 2}+\beta_{c}\right)} d s, & \Re\left(\beta_{c}\right)>0 .\end{cases}
$$

with $\Re \sqrt{1-s^{2}}, \Im \sqrt{1-s^{2}} \geq 0$. The impedance boundary condition satisfied by $G_{\beta_{c}}$ is a consequence of the equation [19]

$$
\text { (3.6) } \frac{\partial \hat{P}_{\beta_{c}}}{\partial \eta}((\xi, \eta))=-i \beta_{c} \hat{P}_{\beta_{c}}((\xi, \eta))-\frac{1}{2} \beta_{c} H_{0}^{(1)}\left(\sqrt{\left(\xi^{2}+\eta^{2}\right)}\right), \quad(\xi, \eta) \in \bar{U} \backslash\{0\} .
$$

It can be seen from (3.5) that $\hat{P}_{\beta_{c}} \in C(\bar{U}) \cap C^{2}(U)$ and satisfies (with $k=1$ ) the Helmholtz equation in $U$. From an alternative representation for $\hat{P}_{\beta_{c}}[19$, equation (21)] we can see that $\hat{P}_{\beta_{c}} \in C^{\infty}(\bar{U} \backslash\{0\})$ and satisfies (with $k=1$ ) the Sommerfeld radiation and boundedness conditions in $U$.

Given $\phi \in C\left(\gamma_{2}\right)$, call the function

$$
w(x)=S^{\beta_{c}} \phi(x)=\int_{\gamma_{2}} G_{\beta_{c}}(x, y) \phi(y) d s(y), \quad x \in \bar{U},
$$

the modified acoustic single-layer potential with density $\phi$. Clearly $w \in C^{\infty}\left(\bar{U} \backslash \gamma_{2}\right)$ and from Remark 3.1 and the above-mentioned properties of $\hat{P}_{\beta_{c}}$ we have the following results. 
LEMma 3.7. The modified single-layer potential $w$ with continuous density $\phi$ is continuous in $\bar{U}$.

LEMma 3.8. The modified single-layer potential $w \in C^{2}\left(\bar{U} \backslash \gamma_{2}\right)$ satisfies the Helmholtz equation (2.2) and the Sommerfeld radiation and boundedness conditions (2.4) in $U$.

Let $C_{0}\left(\gamma_{2}\right):=\left\{\psi \in C\left(\gamma_{2}\right): \psi\left(x^{(j)}\right)=0, j=1,2\right\}$. From Lemmas 3.1 and 3.2 and equation (3.6) we obtain easily the following result.

Lemma 3.9. If $\phi \in C_{0}\left(\gamma_{2}\right)$ the modified single-layer potential $w$ satisfies $w \in$ $\mathcal{R}(U)$ and

$$
\frac{\partial w}{\partial n}(x)-i k \beta_{c} w(x)=\left\{\begin{aligned}
-\phi(x), & x \in \gamma_{2}, \\
0, & x \in \gamma_{3} .
\end{aligned}\right.
$$

In the next theorem and subsequently we abbreviate $S_{\gamma_{j}}$ as $S_{j}$ and $K_{\gamma_{j}}$ as $K_{j}$, for $j=1,2$. The direction of the normals in the definitions of $K_{1}$ and $K_{2}$ are as shown in Figure 5.1. The following is a straightforward extension of Lemma 3.3 - see [20, p. 76]. We distinguish by subscripts + and - limits obtained by approaching $\gamma_{1} \cup \gamma_{2}$ from inside and outside, respectively.

TheOREM 3.10. If $\psi_{1} \in C\left(\gamma_{1}\right), \psi_{2} \in C\left(\gamma_{2}\right)$ and $\psi_{1}\left(x^{(j)}\right)=\psi_{2}\left(x^{(j)}\right)$, for $j=1,2$, then the double-layer potential $K_{1} \psi_{1}(x)-K_{2} \psi_{2}(x)$ can be continuously extended from $\mathbf{R}^{2} \backslash \bar{S}$ to $\mathbf{R}^{2} \backslash S$ and from $S$ to $\bar{S}$ with limiting values $\left(K_{1} \psi_{1}-K_{2} \psi_{2}\right)_{ \pm}(x)= \begin{cases}\left(K_{1} \psi_{1}-K_{2} \psi_{2}\right)(x) \pm \frac{1}{2} \psi_{1}(x), & x \in \gamma_{1} \backslash\left\{x^{(1)}, x^{(2)}\right\}, \\ \left(K_{1} \psi_{1}-K_{2} \psi_{2}\right)(x) \pm \frac{1}{2} \psi_{2}(x), & x \in \gamma_{2} \backslash\left\{x^{(1)}, x^{(2)}\right\}, \\ \left(K_{1} \psi_{1}-K_{2} \psi_{2}\right)(x)+\left(\frac{1}{2} \pm \frac{1}{2}\right) \psi_{2}(x), & x=x^{(1)}, x^{(2)} .\end{cases}$

Define single-layer potential operators $S_{i j}, i, j=1,2$, on $C\left(\gamma_{i}\right)$ by

$$
S_{i j} \phi(x)=\int_{\gamma_{i}} G_{f}(x, y) \phi(y) d s(y), \quad x \in \gamma_{j},
$$

and define double-layer potential operators $K_{i j}, i, j=1,2$, on $C\left(\gamma_{i}\right)$ by

$$
K_{i j} \psi(x)=\int_{\gamma_{i}} \frac{\partial G_{f}(x, y)}{\partial n(y)} \psi(y) d s(y)+(-1)^{j}\left(1-\delta_{i j}\right) \frac{1}{2} \psi(x) E(x), \quad x \in \gamma_{j}
$$


where $E(x)=1, x=x^{(1)}, x^{(2)}$, and $E(x)=0$ otherwise.

Define the modified single-layer potential operator $S_{22}^{\beta_{c}}$ on $C\left(\gamma_{2}\right)$ by

$$
S_{22}^{\beta_{c}} \phi(x)=\int_{\gamma_{2}} G_{\beta_{c}}(x, y) \phi(y) d s(y), \quad x \in \gamma_{2} .
$$

A consequence of Remark 3.1, Lemma 3.7 and Theorem 3.10 is the following result.

TheOREM 3.11. For $i, j=1,2$ the operators $S_{i j}$ and $K_{i j}$ have the mapping properties,

$$
\begin{aligned}
& S_{i j}: C\left(\gamma_{i}\right) \rightarrow C\left(\gamma_{j}\right), \\
& K_{i j}: C\left(\gamma_{i}\right) \rightarrow C\left(\gamma_{j}\right) .
\end{aligned}
$$

Further,

$$
S_{22}^{\beta_{c}}: C\left(\gamma_{2}\right) \rightarrow C\left(\gamma_{2}\right)
$$

In fact each of the mappings in Theorem 3.11 is continuous but we will not need this property subsequently.

4. Reformulation of BVP1 as an integral equation. For simplicity we assume henceforth that $x_{0} \notin \gamma_{2}$, so that $x_{0} \in D \backslash \gamma_{2}$.

THEOREM 4.1. Suppose that p satisfies BVP1. Then

$$
\begin{array}{r}
p(x)=\int_{\gamma_{2}} G_{\beta_{c}}(x, y)\left(i k \beta_{c} p(y)-\frac{\partial p(y)}{\partial n}\right) d s(y)+ \\
\eta\left(x_{0}\right) G_{\beta_{c}}\left(x, x_{0}\right), x \in \bar{U} \backslash\left\{x_{0}\right\},
\end{array}
$$

where

$$
\eta\left(x_{0}\right)= \begin{cases}1, & x_{0} \in U \\ 0, & x_{0} \in S\end{cases}
$$

and

$$
\begin{aligned}
\varepsilon(x) p(x)= & \int_{\gamma_{2}}\left(G_{f}(x, y) \frac{\partial p(y)}{\partial n}-\frac{\partial G_{f}(x, y)}{\partial n(y)} p(y)\right) d s(y)+ \\
& \int_{\gamma_{1}} p(y)\left(\frac{\partial G_{f}(x, y)}{\partial n(y)}-i k \beta(y) G_{f}(x, y)\right) d s(y)+ \\
& \left(1-\eta\left(x_{0}\right)\right) G_{f}\left(x, x_{0}\right), \quad x \in \bar{S} \backslash\left\{x_{0}\right\},
\end{aligned}
$$


where

$$
\varepsilon(x)=\left\{\begin{aligned}
1, & x \in S, \\
\frac{1}{2}, & x \in \partial S \backslash\left\{x^{(1)}, x^{(2)}\right\}, \\
0, & x \in\left\{x^{(1)}, x^{(2)}\right\},
\end{aligned}\right.
$$

and the directions of the normal are as indicated in Figure 5.1.

Proof. We first consider the case $x \in U \backslash\left\{x_{0}\right\}$ and apply Green's 2nd theorem to the functions $u=p$ and $v=G_{\beta_{c}}(x,$.$) in a region E$ consisting of that part of $U$ contained in a large circle of radius $R$ centred on the origin, excluding small circles of radius $\delta$ surrounding $x$ and $x_{0}$. Since $\nabla^{2} u+k^{2} u=\nabla^{2} v+k^{2} v=0$ in $E$, we obtain

$$
\int_{\partial E}\left(u \frac{\partial v}{\partial n}-v \frac{\partial u}{\partial n}\right) d s=0 .
$$

Letting $\delta \rightarrow 0$ and $R \rightarrow \infty$ in (4.3) we obtain, for $x \in U \backslash\left\{x_{0}\right\}$,

(4.4) $p(x)=\eta\left(x_{0}\right) G_{\beta_{c}}\left(x, x_{0}\right)+\int_{\partial U}\left(p(y) \frac{\partial G_{\beta_{c}}(x, y)}{\partial n(y)}-G_{\beta_{c}}(x, y) \frac{\partial p(y)}{\partial n}\right) d s(y)$,

where $\eta\left(x_{0}\right)=1$ for $x_{0} \in U$, and 0 for $x_{0} \in S$. In (4.4) the terms $p(x)$ and $G_{\beta_{c}}\left(x, x_{0}\right)$ are the limits as $\delta \rightarrow 0$ of that part of the integral in (4.3) around the small circles surrounding $x$ and $x_{0}$ respectively. The part of the integral on the circular arc of radius $R$ in (4.3) vanishes as $R \rightarrow \infty$ since $u$ and $v$ both satisfy the Sommerfeld radiation and boundedness conditions (2.4).

Utilising the boundary condition satisfied by $p$ on $\partial U$ (equation $(2.3)$ ) and by $G_{\beta_{c}}$ on $\partial U$ we obtain equation (4.1) for $x \in U \backslash\left\{x_{0}\right\}$. Using the continuity of $p$ and that of the modified single layer potential (Lemma 3.7) we extend the validity of (4.1) from $U \backslash\left\{x_{0}\right\}$ to $\bar{U} \backslash\left\{x_{0}\right\}$.

To obtain equation (4.2) for $x \in \mathbf{R}^{2} \backslash \partial S$ we proceed similarly, choosing $R>$ $\max _{y \in \gamma_{1}}|y|,|x|,\left|x_{0}\right|$, and applying Green's second theorem first in $U \cap B_{R}$ and then in $D \cap B_{R}$, but excluding in each case small circles of radius $\delta$ surrounding $x$ and $x_{0}$. (These applications are valid since $P \in \mathcal{R}(D) \cap \mathcal{R}(U) \cap C^{1}\left(\bar{D} \backslash \gamma_{1}\right)$. ) Letting $\delta \rightarrow 0$, subtracting the resulting equations, and utilising the boundary condition (2.3) satisfied by $p$ on $\gamma_{1}$ we obtain equation (4.2) for $x \in S \backslash\left\{x_{0}\right\}$. Using the continuity of 
$p$ in $\bar{D} \backslash\left\{x_{0}\right\}$ and of $\partial p / \partial n$ on $\partial D$ and $\partial U$ we extend the validity of (4.2) from $S \backslash\left\{x_{0}\right\}$ to $\bar{S} \backslash\left\{x_{0}\right\}$ via Lemma 3.1 and Theorem 3.10 .

Equations (4.1) and (4.2) express the pressure in $D$ in terms of the unknowns $p$ and $\partial p / \partial n$ on $\gamma_{2}$ and $p$ on $\gamma_{1}$. Let $p_{1}:=\left.p\right|_{\gamma_{1}}, p_{2}:=\left.p\right|_{\gamma_{2}}$ and $q:=i k \beta_{c} p_{2}-\left.(\partial p / \partial n)\right|_{\gamma_{2}}$. The values of $q$ at the end-points of $\gamma_{2}$ are zero since $\partial p / \partial n \in C(\partial U)$ and $\partial p / \partial n=i k \beta_{c} p$ on $\gamma_{3}$. In terms of the integral operators defined in section 3 , Theorem 4.1 shows that $p_{1}, p_{2}$ and $q$ satisfy the following integral equation problem:

IEP1 Find $p_{1} \in C\left(\gamma_{1}\right), p_{2} \in C\left(\gamma_{2}\right)$ and $q \in C_{0}\left(\gamma_{2}\right)$ such that

$$
\begin{aligned}
p_{2} & =S_{22}^{\beta_{c}} q+g_{\beta_{c}}, \\
\frac{1}{2} p_{2} & =K_{12} p_{1}-i k S_{12}\left(\beta p_{1}\right)+i k \beta_{c} S_{22} p_{2}-S_{22} q+g_{2}, \\
\frac{1}{2} p_{1} & =K_{11} p_{1}-i k S_{11}\left(\beta p_{1}\right)+i k \beta_{c} S_{21} p_{2}-S_{21} q-K_{21} p_{2}+g_{1},
\end{aligned}
$$

where $g_{\beta_{c}}, g_{2} \in C\left(\gamma_{2}\right), g_{1} \in C\left(\gamma_{1}\right)$ are defined by $g_{\beta_{c}}(x):=\eta\left(x_{0}\right) G_{\beta_{c}}\left(x, x_{0}\right), g_{2}(x):=$ $\left(1-\eta\left(x_{0}\right)\right) G_{f}\left(x, x_{0}\right), x \in \gamma_{2}$, and $g_{1}(x):=\left(1-\eta\left(x_{0}\right)\right) G_{f}\left(x, x_{0}\right), x \in \gamma_{1}$.

The next theorem, our main result, shows that, conversely, if $p_{1}, p_{2}$, and $q$ satisfy IEP1 and $p$ is defined by (4.1) and (4.2) then $p$ satisfies BVP1. As a corollary of this theorem and the uniqueness result, Theorem 2.1, we have immediately that IEP1 has at most one solution for all wavenumbers $k>0$.

THEOREM 4.2. If $p_{1}, p_{2}$ and $q$ satisfy IEP 1 and $p: \bar{D} \backslash\left\{x_{0}\right\} \rightarrow \mathbf{C}$ is defined by

$$
\begin{array}{rlrl}
p(x)= & S^{\beta_{c}} q(x)+\eta\left(x_{0}\right) G_{\beta_{c}}\left(x, x_{0}\right), & & x \in \bar{U}, \\
\varepsilon(x) p(x)= & K_{1} p_{1}(x)-i k S_{1}\left(\beta p_{1}\right)(x)+i k \beta_{c} S_{2} p_{2}(x)-S_{2} q(x) & \\
& -K_{2} p_{2}(x)+\left(1-\eta\left(x_{0}\right)\right) G_{f}\left(x, x_{0}\right), & x \in \bar{S} \backslash \gamma_{2},
\end{array}
$$

then $p$ satisfies BVP1, and $p_{1}=\left.p\right|_{\gamma_{1}}, p_{2}=\left.p\right|_{\gamma_{2}}$ and $q=i k \beta_{c} p_{2}-\partial p /\left.\partial n\right|_{\gamma_{2}}$.

Proof. With $p$ defined by (4.8) and (4.9), define $P$ by (2.1), i.e. $P(x)=p(x)-$ $G_{f}\left(x, x_{0}\right), x \in \bar{D} \backslash\left\{x_{0}\right\}$. The proof is split into several steps.

Step I. We show that $P \in C^{2}\left(\bar{U} \backslash \gamma_{2}\right) \cap \mathcal{R}(U)$, that $p$ satisfies the Sommerfeld radiation and boundedness conditions, that $p$ is continuous on $\partial D$, and that $p_{2}=\left.p\right|_{\gamma_{2}}$, $p_{1}=\left.p\right|_{\gamma_{1}}, q=i k \beta_{c} p_{2}-\partial p /\left.\partial n\right|_{\gamma_{2}}$ and $\partial p / \partial n=i k \beta_{c} p$ on $\gamma_{3}$. 
With $p$ defined by (4.8), $\left.p\right|_{\gamma_{2}}=p_{2}$ follows immediately from (4.5). By Lemma 3.8 it is clear from (4.8) that $p$ satisfies the Sommerfeld radiation and boundedness conditions (2.4) and, by Lemmas 3.8 and 3.9, that $P \in C^{2}\left(\bar{U} \backslash \gamma_{2}\right) \cap \mathcal{R}(U)$. From Lemma 3.9 and (4.5) the value of the normal derivative is

$$
\frac{\partial p}{\partial n}= \begin{cases}i k \beta_{c} p_{2}-q, & \text { on } \gamma_{2} \\ i k \beta_{c} p, & \text { on } \gamma_{3}\end{cases}
$$

Observe from (4.6) and (4.7) that $p_{1}\left(x^{(j)}\right)=p_{2}\left(x^{(j)}\right)$, for $j=1,2$ (note that $K_{21} p_{2}\left(x^{(j)}\right)=0$, for $\left.j=1,2\right)$. With $p$ defined by (4.9) that $p(x)=p_{1}(x)$ for $x \in \gamma_{1} \backslash\left\{x^{(1)}, x^{(2)}\right\}$ is immediate from (4.7). Since $p_{1} \in C\left(\gamma_{1}\right)$ it follows that $p$ is continuous on $\partial D$ and that $p_{1}=\left.p\right|_{\gamma_{1}}$.

Step II. Next, we show that the function defined by the right hand side of (4.9) is identically zero in $\mathbf{R}^{2} \backslash \bar{S}$.

Let $\phi: \mathbf{R}^{2} \backslash S \rightarrow \mathbf{C}$ be defined by

$$
\phi(x):= \begin{cases}K_{1} p_{1}(x)-i k S_{1}\left(\beta p_{1}\right)(x)+i k \beta_{c} S_{2} p_{2}(x)-S_{2} q(x) & \\ -K_{2} p_{2}(x)+\left(1-\eta\left(x_{0}\right)\right) G_{f}\left(x, x_{0}\right), & x \in \mathbf{R}^{2} \backslash \bar{S} \\ 0, & x \in \partial S .\end{cases}
$$

From Lemma 3.6 it follows that $\phi \in C^{2}\left(\mathbf{R}^{2} \backslash \bar{S}\right)$ and satisfies in $\mathbf{R}^{2} \backslash \bar{S}$ the Helmholtz equation and the Sommerfeld radiation and boundedness conditions. We shall show that $\phi \in C\left(\mathbf{R}^{2} \backslash S\right)$ and hence $\phi \equiv 0$ by Theorem 2.2 .

To see that $\phi \in C(\bar{U})$, let $x \rightarrow y \in \gamma_{2}$, with $x \in U$. By Theorem 3.10 and Remark 3.1 ,

$$
\begin{aligned}
\phi(x) & \rightarrow\left(K_{12} p_{1}-i k S_{12}\left(\beta p_{1}\right)+i k \beta_{c} S_{22} p_{2}-S_{22} q\right)(y)+\left(1-\eta\left(x_{0}\right)\right) G_{f}\left(y, x_{0}\right)-\frac{1}{2} p_{2}(y) \\
& =0
\end{aligned}
$$

by (4.6). Similarly, let $x \rightarrow y \in \gamma_{1}$, with $x \in \mathbf{R}^{2} \backslash \bar{D}$. By Theorem 3.10 and Remark 3.1 ,

$$
\phi(x) \rightarrow\left(K_{11} p_{1}-i k S_{11}\left(\beta p_{1}\right)+i k \beta_{c} S_{21} p_{2}-S_{21} q-K_{21} p_{2}\right)(y)+
$$




$$
\begin{aligned}
& \left(1-\eta\left(x_{0}\right)\right) G_{f}\left(y, x_{0}\right)-\frac{1}{2} p_{1}(y) \\
= & 0
\end{aligned}
$$

by (4.7). Thus $\phi \in C\left(\mathbf{R}^{2} \backslash S\right)$.

Step III. We now introduce a $C^{2}$-contour $\Gamma$ with end-points $x^{(1)}$ and $x^{(2)}$ as shown in Figure 5.2. The key features of $\Gamma$ are that it lies in $\bar{U}$, that $\Gamma \cap \gamma_{2}=$ $\left\{x^{(1)}, x^{(2)}\right\}$, that $\Gamma$ coincides with $\partial U$ near its end-points $x^{(1)}$ and $x^{(2)}$ and that $x_{0}$ lies inside $\Gamma \cup \gamma_{1}$. Let $D_{j}, j=1,2$, denote the $C^{2}$ region which is the interior of $\Gamma \cup \gamma_{j}$.

We may apply Green's representation theorem [15] to $p$ in $D_{2}$ and use Lemma 3.1 and Lemma 3.3 to obtain that

$$
\begin{aligned}
& \varepsilon_{1}(x) p(x)=\int_{\partial D_{2}}\left(\frac{\partial G_{f}(x, y)}{\partial n(y)} p(y)-G_{f}(x, y) \frac{\partial p(y)}{\partial n}\right) d s(y)+ \\
& \eta\left(x_{0}\right) G_{f}\left(x, x_{0}\right), \quad x \in \mathbf{R}^{2} \backslash\left\{x_{0}\right\},
\end{aligned}
$$

where

$$
\varepsilon_{1}(x):=\left\{\begin{aligned}
1, & x \in D_{2} \\
1 / 2, & x \in \partial D_{2} \\
0, & x \in\left(\mathbf{R}^{2} \backslash \bar{D}_{2}\right) .
\end{aligned}\right.
$$

Step $I V$. We finish the proof by recovering the regularity properties of $p$ in $S$ and the boundary condition on $\gamma_{1}$ and by showing that $p$ satisfies the Helmholtz equation.

For $x \in \mathbf{R}^{2} \backslash\left(S \cup \gamma_{1}\right)$ add equation (4.12) to equation (4.11) (noting that $\phi \equiv 0$ in this region by Step II) and, for $x \in S \cup \gamma_{1}$, add equation (4.12) to equation (4.9). Using the boundary conditions (4.10) recovered in Step I we obtain

$$
\begin{aligned}
\varepsilon_{2}(x) p(x)= & \int_{\partial D_{1}}\left(\frac{\partial G_{f}(x, y)}{\partial n(y)} p(y)-G_{f}(x, y) a(y)\right) d s(y) \\
& +G_{f}\left(x, x_{0}\right), \quad x \in \mathbf{R}^{2}
\end{aligned}
$$

where

$$
\varepsilon_{2}(x):= \begin{cases}1, & x \in D_{1}, \\ 1 / 2, & x \in \partial D_{1}, \\ 0, & x \in \mathbf{R}^{2} \backslash \bar{D}_{1},\end{cases}
$$


and

$$
a(x):= \begin{cases}\frac{\partial p}{\partial n}(x), & x \in \Gamma, \\ i k \beta_{c} p(x), & x \in \gamma_{1} \backslash\left\{x^{(1)}, x^{(2)}\right\} .\end{cases}
$$

From (4.10) and the continuity of $p$ on $\partial D$ it follows that $a \in C\left(\partial D_{1}\right)$. In operator form we may rewrite equation (4.14) as

$$
\varepsilon_{2}(x) p(x)=\left(K_{\partial D_{1}} p\right)(x)-\left(S_{\partial D_{1}} a\right)(x)+G_{f}\left(x, x_{0}\right), \quad x \in \mathbf{R}^{2} .
$$

Now, $S_{\partial D_{1}} a \in \mathcal{R}\left(\mathbf{R}^{2} \backslash \bar{D}_{1}\right)$ from Lemma 3.2 , which implies immediately that $K_{\partial D_{1}} p \in$ $\mathcal{R}\left(\mathbf{R}^{2} \backslash \bar{D}_{1}\right)$; and that $K_{\partial D_{1}} p \in \mathcal{R}\left(D_{1}\right)$ follows from Lemma 3.5. Since also $S_{\partial D_{1}} a \in$ $\mathcal{R}\left(D_{1}\right)$ it follows that $P \in \mathcal{R}\left(D_{1}\right)$. Further, from Lemma 3.6, $P \in C^{2}\left(D_{1}\right)$ and satisfies the Helmholtz equation in $D_{1}$. The impedance boundary condition on $\gamma_{1}$ is retrieved via Lemmas 3.2 and 3.5 which give that

$\frac{\partial S_{\partial D_{1}} a(x)_{+}}{\partial n}-\frac{\partial S_{\partial D_{1}} a(x)_{-}}{\partial n}=a(x), \quad \frac{\partial K_{\partial D_{1}} p(x)_{+}}{\partial n}=\frac{\partial K_{\partial D_{1}} p(x)_{-}}{\partial n}, \quad x \in \gamma_{1}$. Since $D_{1} \subset D$ may be arbitrarily large we have completed the proof.

5. Conclusions. In this paper the problem of acoustic scattering from a source within a a disturbance of arbitrary cross-section and surface impedance out onto a homogeneous impedance plane has been formulated as a boundary value problem for the Helmholtz equation and then reformulated as a coupled system of three boundary integral equations. Equivalence of the boundary value problem and integral equation formulation at all wavenumbers has been demonstrated so that uniqueness of solutions is ensured. Hence the formulation does not suffer from irregular frequencies often encountered in numerical treatment of scattering problems.

\section{REFERENCES}

[1] L.-S. HWANG \& E.O. TUCK, On the oscillations of harbours of arbitrary shape, J. Fluid Mech. 42 (1970), Vol 42(3), 447-464.

[2] J.-J. LEE, Wave-induced oscillations in harbours of arbitrary geometry, J. Fluid Mech. 45 (1971) 372-394. 
[3] R.P. SHAW, Long period forced harbor oscillations, Topics in Ocean Engineering (ed. C.I. Bretschneider; Gulf Publishing Company, Texas, 1971).

[4] O. GARTMEIER, Eine Integralgleichungsmethode für akustiche Reflexionsprobleme au lokal gestörten Ebenen, Math Meth. Appl. Sciences 3 (1981) 128-144.

[5] S.N. CHANDLER-WILDE, D.C. HOTHERSAlL, D.H. CROMBIE \& A.T. PEPLOW, Efficiency of an acoustic screen in the presence of an absorbing boundary, In Rencontres Scientifiques du Cinquantenaire: Ondes Acoustiques et Vibratoires, Interaction FluideStructures Vibrantes, Publication du CNRS Laboratoire de Mécanique et d'Acoustique 126 (Marseille, 1991).

[6] D.C. HOThERSALL, S.N. CHANDLER-WILDE, \& N.M. HAJMIRZAE, Efficiency of single noise barriers, J. Sound and Vibration 148 (1991) 365-380.

[7] A.T. PEPLOW, \& S.N. CHANDLER-WILDE, Noise propagation from a cutting of arbitrary cross-section and impedance, J. Sound and Vibration 223 (1999) 355-378.

[8] A. WILLERS, The Helmholtz equation in disturbed half-spaces, Math Meths in the Appl. Sciences 9 (1987) 312-323.

[9] S.-K. JENG \& S.-T. TZENG, Scattering from a cavity-backed slit in a ground plane - TM case, IEEE Trans. Antenn. Propagat. 39 (1991) 1598-1604.

[10] J.-M. JIN \& J.-L. VOLAKIS, Electromagnetic scattering by and transmission through a 3dimensional slot in a thick conducting plane, IEEE Trans. Antenn. Propagat. 39 (1991) 97-104.

[11] T.-M. WANG \& H. LING, Electromagnetic scattering from 3-dimensional cavities via a connection scheme, IEEE Trans. Antenn. Propagat. 39 (1991) 1505-1513.

[12] J.S. ASVESTAS \& R.E. KLEINMAN, Electromagnetic scattering by indented screens, IEEE Trans. Antenn. Propagat. 41 (1994) 22-30.

[13] P.A. KRUTITSKII, Wave scattering in a 2-D exterior domain with cuts: The Neumann problem, Z. Angew. Math. Mech. 80 (2000) 535-546.

[14] P.A. KRUTITSKII, The oblique derivative problem for the Helmholtz equation and scattering tidal waves, Proc. Roy. Soc. London Ser. A 457 (2001) 1735-1755.

[15] D. COLTON \& R. KRESS, Integral Equation Methods in Scattering Theory (WileyInterscience Publication 1983).

[16] F. RELLICH, Uber das asymptotische Verhallen der Lösungen von $\nabla^{2} u+\lambda u=0$ in unendlichen GebeitenJber, Deutsch. Math. Verein 53 (1943) 57-65.

[17] D. COLTON \& R. KRESS, Inverse Acoustic and Electromagnetic Scattering (Springer-Verlag 1992).

[18] K. JÖRGENS, Linear Integral Operators, (Pitman 1982).

[19] S.N. CHANDLER-WILDE \& D.C. HOTHERSALL, Efficient calculation of the Green- 
function for acoustic propagation over a homogeneous impedance plane, J. Sound and Vibration 180 (1995) 705-724.

[20] R. KRESS, Linear Integral Equations (Springer-Verlag 1990). 


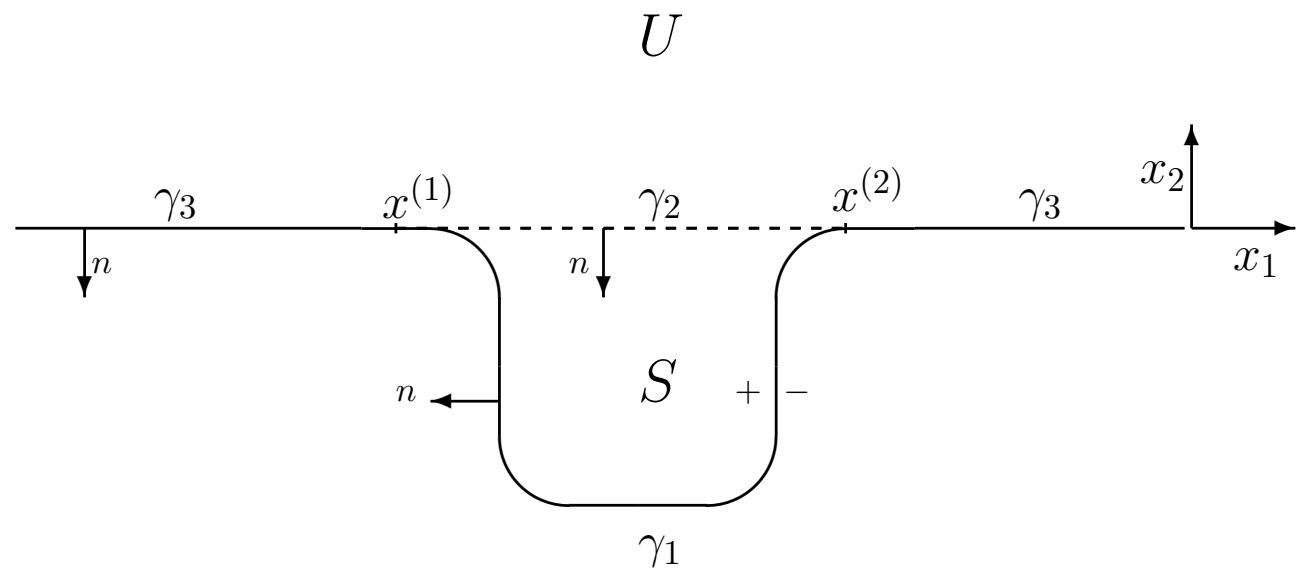

FIG. 5.1. Geometry of the model 


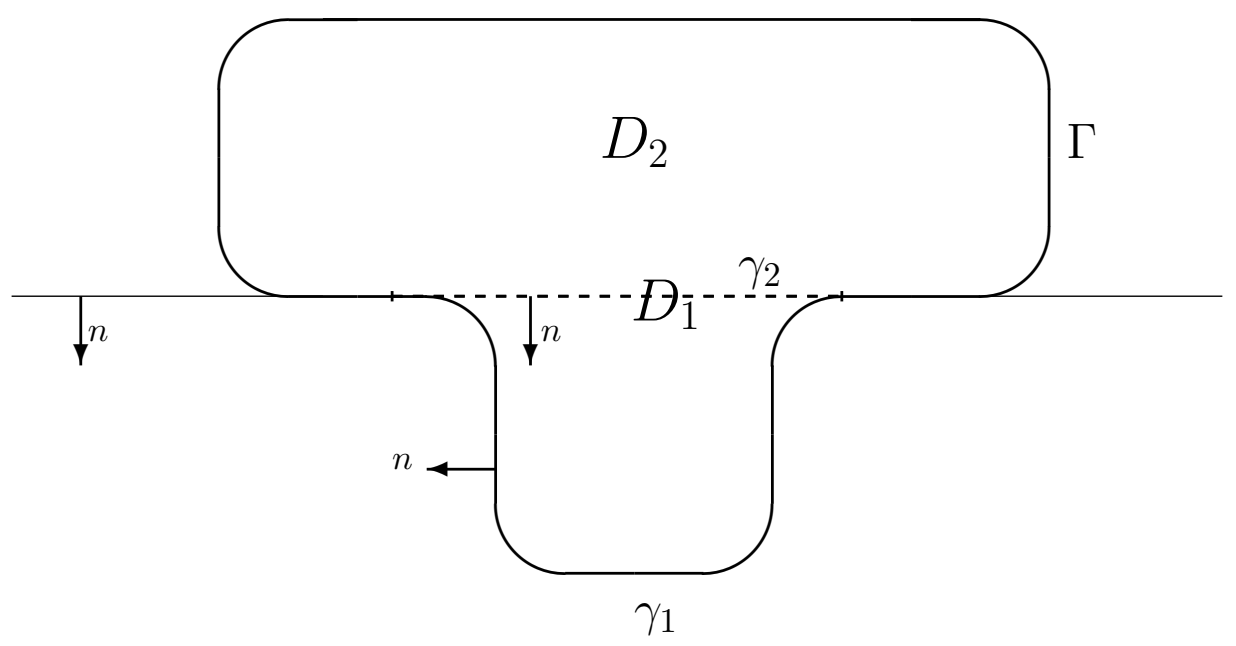

FIG. 5.2. Modified region 